\title{
Why does my patient's basilar artery continue to grow? A four-year case study of a patient with symptoms of vertebrobasilar dolichoectasia
}

\author{
Dao Pei Zhang ${ }^{1,2+}$, Yan Fang Peng ${ }^{2 \dagger}$, Qian Kun Ma ${ }^{3}$, Min Zhao ${ }^{1 *}$, Huai Liang Zhang ${ }^{1 *}$ and Suo Yin ${ }^{4}$
}

\begin{abstract}
Background: Vertebrobasilar dolichoectasia (VBD) is a clinical entity associated with ischemic stroke, compression of cranial nerves or brainstem, and hydrocephalus. There have been relatively few studies following the progression of VBD in patients presenting with a variety of diverse clinical features.

Case presentation: Here, we report a case study of a male with progressive VBD who was followed from November 2012 to December 2016. The patient had diagnosed hypertension for several years and suffered from left peripheral facial paralysis, recurrent ischemic attacks in the brainstem and cerebellum, obstructive hydrocephalus and frequent pneumonia. A series of cranial $C T$ and multi-modal MRI scans were performed to explore the brain imaging features of the patient during follow-up.
\end{abstract}

Conclusions: The presented case study suggests that aging, uncontrolled hypertension, arterial dissection and infection may contribute to the exacerbation of VBD and recurrent ischemic stroke.

Keywords: Vertebrobasilar dolichoectasia, Obstructive hydrocephalus, Stroke, Artery dissection, Magnetic resonance imaging

\section{Background}

Vertebrobasilar dolichoectasia (VBD) is a rare disease characterized by expansion, elongation, and tortuosity of the vertebrobasilar arteries [1]. Though the prevalence of VBD has been estimated to be in the range of 0.2-4. $4 \%$ for the general population and $2.6-17.1 \%$ for stroke patients [2], reliable population-based data are lacking. Complications from VBD include transient ischemic attack, compression of brainstem or cranial nerves, ischemic stroke, subarachnoid hemorrhage, and obstructive hydrocephalus $[1,3]$. A previous systematic review of 375 patients concluded that patients with VBD are at an elevated risk of neurological deterioration during a 5year follow-up [2].

\footnotetext{
*Correspondence: byts1969@126.com; zhl121@126.com

${ }^{\dagger}$ Equal contributors

${ }^{1}$ Department of Neurology, The First Affiliated Hospital of Henan University

of CM, Zhengzhou, China

Full list of author information is available at the end of the article
}

Generally, VBD has is considered to be a progressive clinical entity that leads to heightened morbidity and mortality [4]. However, few studies have included patients exhibiting diverse clinical features with an elaborate evolving process of VBD. Furthermore, the factors that contribute to VBD growth remain unclear.

The current paper reports the case of a 52-year old man with hypertension who suffered from a series of symptoms over four years, including peripheral facial paralysis, recurrent ischemic stroke attacks affecting subtentorial areas, and obstructive hydrocephalus. Importantly, the patient presented with a gradually growing VBD, slow blood flow, basilar artery (BA) vessel wall hematoma, and vertebral dissection revealed by multimodal magnetic resonance imaging (MRI). Here we describe VBD progression and growth and discuss possible contributing factors.

(C) The Author(s). 2018 Open Access This article is distributed under the terms of the Creative Commons Attribution 4.0 International License (http://creativecommons.org/licenses/by/4.0/), which permits unrestricted use, distribution, and reproduction in any medium, provided you give appropriate credit to the original author(s) and the source, provide a link to the Creative Commons license, and indicate if changes were made. The Creative Commons Public Domain Dedication waiver (http://creativecommons.org/publicdomain/zero/1.0/) applies to the data made available in this article, unless otherwise stated. 


\section{Case presentation}

The presented patient developed left side peripheral facial paralysis in November 2012. A cranial computed tomography (CT) scan showed an elongated and dilated BA, of high density without contrast enhancement, that passed upward to enter the floor of the third ventricle (Fig. 1a). A head CT angiography later verified a diagnosis of VBD (CT imaging data are not available).

In August 2013, the patient was hospitalized for three days due to dizziness, weakness and numbness of the left limbs, and alalia. Neurological examinations revealed loss of muscle strength [grade 3 on the Medical Research Council (MRC) scale] and moderate loss of pinprick and temperature sensation in the left limbs without involvement of the face. A CT scan showed a right pontine infarction and the same elongated BA that was seen in November 2012 (Fig. 1b). The patient declined multi-modal MRI.

The patient was again hospitalized for two days in January 2015 due to dizziness and numbness of the right limbs with bucking and alalia. Neurological examination revealed dysarthria, dysphagia, severe right hemiplegia, with muscle strength of the upper limb at a grade 2 and the lower limb at a grade 3 (MRC) and loss of pinprick sensation in the right limbs. Diffusion-weighted imaging (DWI) showed acute ischemic infarction in the mesencephalon-pontine junction (Fig. 2a and b). Threedimensional time-of-flight $\mathrm{MR}$ angiography imaging (TOF-MRA) revealed a dilated, tortuous, and elongated vertebral artery and BA (Fig. 2c). Dynamic susceptibility contrast-enhanced perfusion weighted imaging (DSCPWI) showed decreased cerebral blood volume (CBV) and cerebral blood flow (CBF) in the medulla oblongata, cerebellum, and pons, and elongated mean transition time (MTT), and time to peak (TTP) in the left cerebellum, revealing multiple relatively ischemic territories of the posterior circulation (Fig. 2d-g).

In September 2015, the patient's symptoms worsened, and he was again hospitalized in a somnolent state with a Glasgow Coma Scale score of 12 (eye opening score = 3 , verbal response score $=4$, and motor response score $=$ 5). Neurological examination showed bilateral paralysis with MRC grade 0 , grade 3 , and grade 4 muscle strength of the right limbs, left upper limb, and left lower limb, respectively. Non-enhanced and contrast-enhanced MRI and MRA showed obstructive hydrocephalus due to the growth of the BA (with a double lumen sign suggesting dissection), resulting in the compression of the foramen of Monro (Fig. 3a-d). An emergency ventriculoperitoneal shunt surgery was performed. One month later, reexamination by $\mathrm{CT}$ demonstrated that the hydrocephalus had decreased (Fig. 1c-d). The patient was discharged from the hospital after exhibiting recovery of his limb muscle strength during his hospital stay, at which time he still required supportive care in his daily life.

In December 2016, the patient was wheel chair-bound and complained of dizziness and dysphagia. He was thus hospitalized again. Neurological examinations revealed hypoactive pharyngeal reflex, dysphagia, dysarthria, internuclear ophthalmoplegia, ataxia and paralysis of the right upper and lower limbs. Acute multiple spot-like infarctions (artery-to-artery embolism) involving the left medulla oblongata, cerebellar hemisphere and pons were found through DWI (Fig. $2 \mathrm{~h}$ and i). In addition, the right vertebral artery was not visible (Fig. 2j). High-resolution MRI showed a double lumen of the BA (Fig. 2o). Compared to TOF-MRA imaging, basi-parallel anatomic scanning [5] showed a greater BA diameter and a visible right vertebral artery, suggesting dissection (Fig. 2p). PWI revealed hypo-perfusion of the medulla oblongata, cerebellar hemispheres, and pons (Fig. 2k-n). After approximately 20 days of antiplatelet therapy, the patient was discharged in a severely disabled condition characterized by bed-bound paralysis with MRC grade 1 muscle strength of the right upper limb and both lower limbs, requiring continuous care and attention.

In September 2015 and December 2016, fluid attenuated inversion recovery (FLAIR) vascular hyperintensity
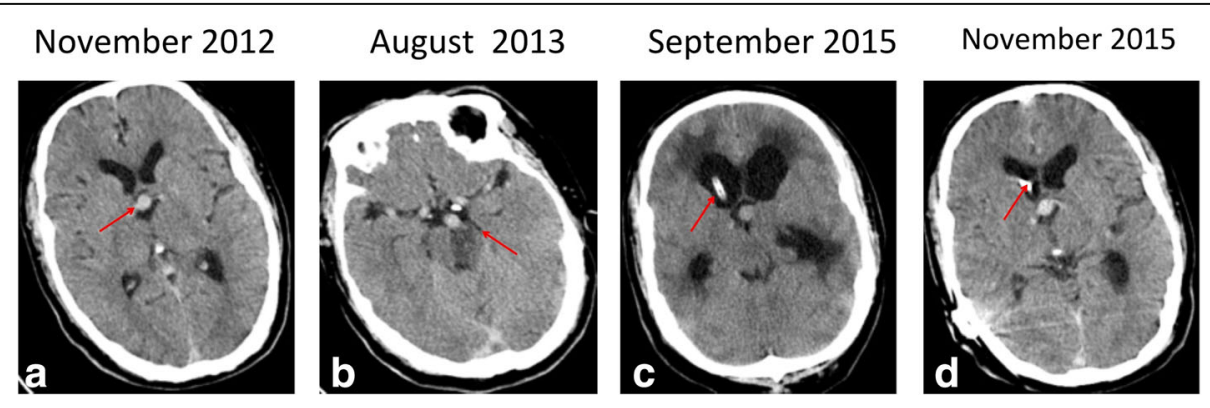

Fig. 1 Serial axial non-enhanced CT scans. (a) Upward displacement of the third ventricle (red arrow) by a markedly ectatic BA. (b) Left pontine infarction compressed by the ectatic BA (red arrow). (c) Bilateral dilated ventricles and low density lesions anterior and around the lower horns on the second day after ventriculoperitoneal shunt placement (red arrow). (d) Reduced ventricular dilation and disappearance of low density lesions around the ventricle horn (red arrow) one month after shunt placement 

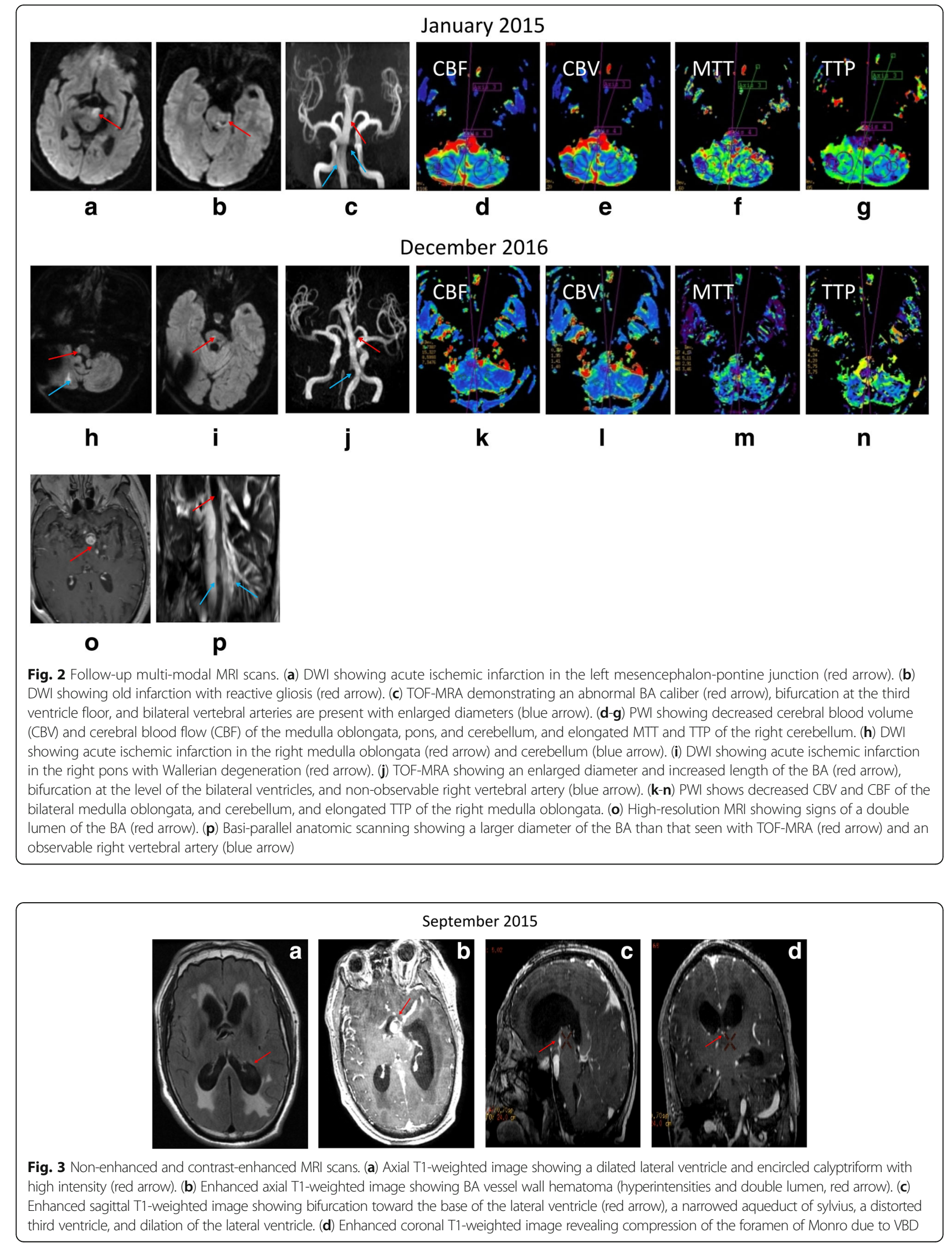
(FVH 3) [6] around the rim of the BA wall indicated slow blood velocity (Fig. 4a and b). In January 2015, TOF-MRA revealed a tortuous, elongated (length, $40.6 \mathrm{~mm}$ ), and dilated (diameter, $8.3 \mathrm{~mm}$ ) BA. In December 2016, TOFMRA revealed greater tortuosity, further elongation (length, $46.9 \mathrm{~mm}$ ), and further dilation (diameter, 10. $5 \mathrm{~mm}$ ) of the BA (Fig. 5a-d). Unfortunately, in March 2017, the patient died of pneumonia secondary to recurrent aspiration due to lower cranial nerve deficits.

\section{Discussion and conclusions}

The diagnosis of BA ectasia is defined by dilation reaching $>4.5 \mathrm{~mm}$ in diameter at the mid-pons level [7]. An increase in BA diameter by $\geq 2 \mathrm{~mm}$ from the baseline diameter or an increase in lateral displacement or height of BA bifurcation is classified as a progression of VBD [8]. In this case, serial TOF-MRA studies conducted almost 2 years apart (January 2015 to December 2016) revealed increases in BA tortuosity, elongation $(40.6 \mathrm{~mm}$ to $46.9 \mathrm{~mm}$ ), and dilation $(8.3 \mathrm{~mm}$ to $10.5 \mathrm{~mm})$. BA dolichoectasia rupture risk has been shown to be associated with an initial artery diameter larger than $10 \mathrm{~mm}$ [9]. Alternative treatments, such as blood pressure reduction therapy, surgery, or endovascular procedures, may be considered in cases of BA enlargement (BA diameter $\geq 10 \mathrm{~mm}$ ) [9]. It is of critical importance to follow the development and progression of patients with VBD so as to implement a proper and timely intervention to improve the patient's prognosis [10].

A recent case report followed a 60-year-old Caucasian man with a 31-year history of secondary progressive multiple sclerosis and uncontrolled arterial hypertension. The report presented a series of images over many years demonstrating the progression of a normal BA becoming dolichoectatic, followed by the formation of an aneurysm [11]. Here, we present a four-year follow-up case of a male with unilateral facial paralysis that was indefinitely associated with VBD, who showed gradual

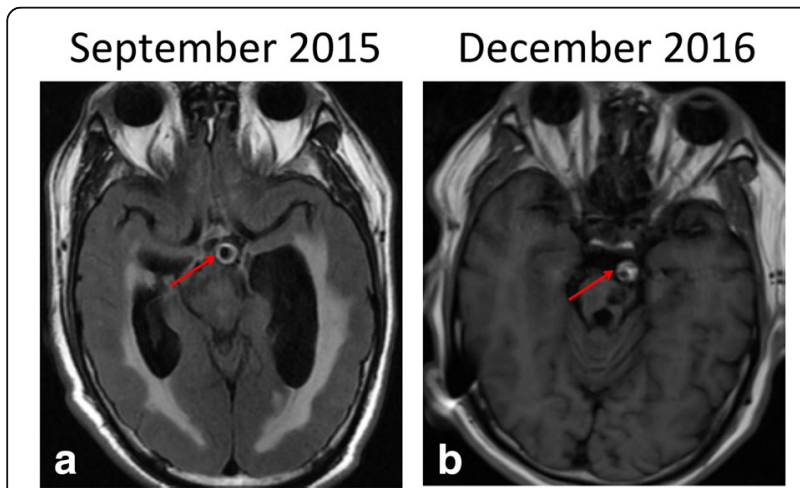

Fig. $4 \mathrm{MRI}-\mathrm{FLAIR}$ scans. (a) A hyperintense rim near the vessel wall (FVH grade 2). (b) A hyperintense signal that nearly fills the entire BA lumen (FVH grade 3) development of recurrent ischemic stroke and obstructive hydrocephalus. The maximum diameter and length of his BA, as revealed by MRA, increased gradually. Moreover, the lateral height of BA bifurcation was increased from the level of the interpeduncular cistern or the suprasellar cistern to the level of the third and lateral ventricles.

The normal blood flow velocity profile over a longitudinal section is parabolic with lowest velocities typically observed near the vessel wall. The blood flow in a patient with VBD is turbulent and slow, causing hypoperfusion, as displayed on PWI [10]. Perfusion images in the current case study, showed hypoperfusion in the blood supply area of VBD. Moreover, a prior study indicated that FVH may demonstrate decreased blood flow velocity in patients with VBD [9]. The presence and extent of $\mathrm{FVH}$ in the BA is classified into three grades and the current patient reached grade 3 , at which time the hyper-intense signal filled the entire lumen. Reduced blood flow can cause hypoperfusion, leading to ischemic symptoms. The recurrent strokes experienced by the presented patient may be partially associated with abnormal VBD hemodynamics. The patient's latest spot-like infarction might have been caused by artery-to-artery embolism and hypoperfusion. However, progressive enlargement of the BA may also pull or compress the origin of the perforating artery, leading to infarction of the blood supply territory [9]. Because DWI showed acute multiple spot-like infarctions involving the left medulla oblongata, cerebellar hemisphere, and pons, the latter mechanism was ruled out in our patient.

Although VBD-associated recurrent ischemic events were observed, the plan for secondary prevention was poor [12]. The general inadequacy of the literature related to VBD-associated stroke treatment do not provide an avenue for high-confidence treatment planning based on guidelines [2]. Aspirin and anticoagulants are an option, but would increase intracerebral bleeding risk [13]. Therefore, we pursued cautious antiplatelet therapy in this case. The efficacy of surgical and radiological interventions should be determined in future studies.

Imaging data confirmed a BA vessel-wall hematoma and dissection of the right vertebral artery. Vertebrobasilar artery dissection may contribute to the deterioration of symptomatic VBD. Furthermore, based on findings indicating that VBD is related to arterial dissection and the presence of a vessel wall hematoma, the authors of a previous study suggested that the two pathologies may share etiologies [14]. Additionally, abnormal hemodynamic stress may play an important role in arterial dissection and vessel wall hematoma formation $[15,16]$. Thus, vertebrobasilar artery dissection may accelerate the progression of VBD [15]. Importantly, vessel wall hematoma development is also a crucial step in the pathological 

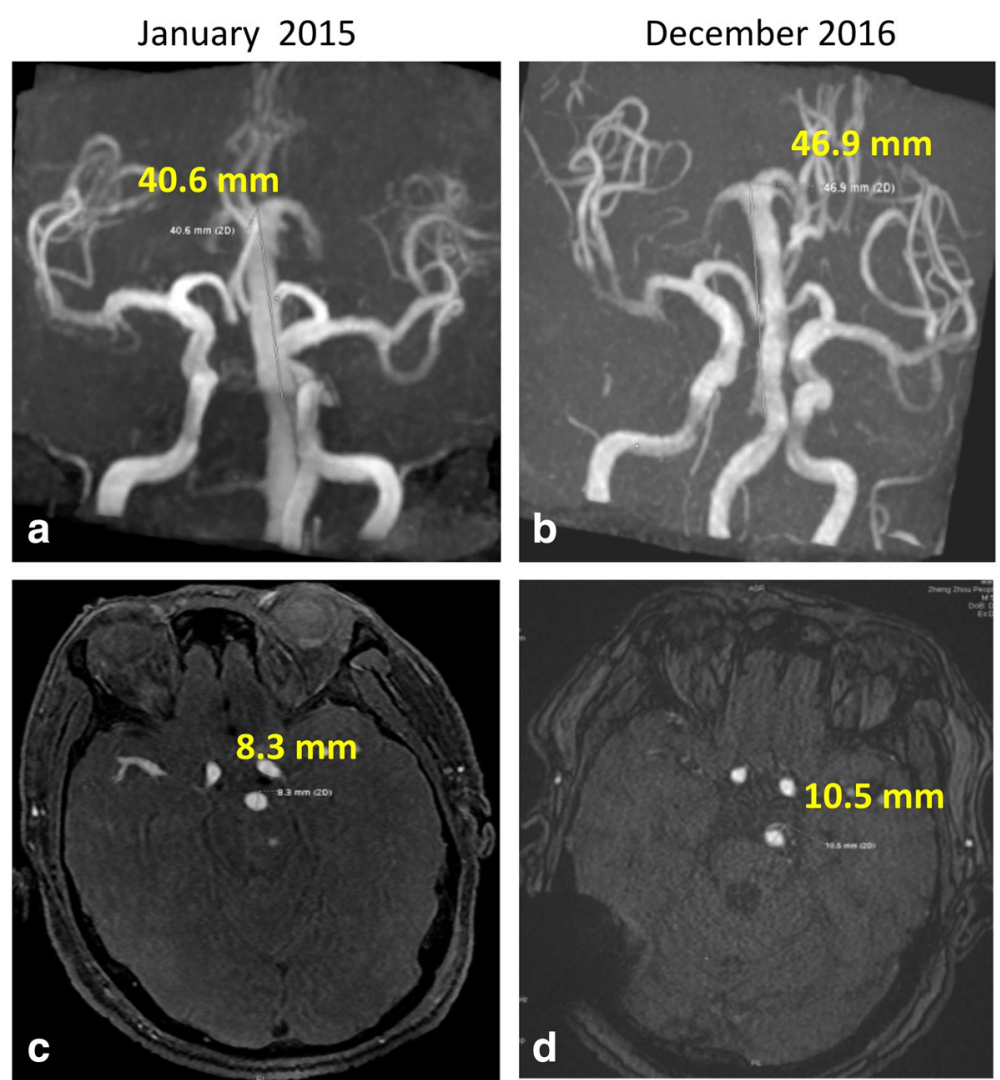

Fig. 5 TOF-MRA scans with BA growth images. (a, c) TOF-MRA images from January 2015 revealing a tortuous and elongated BA (a: length, $40.6 \mathrm{~mm}$ ) with dilation (c: diameter, $8.3 \mathrm{~mm}$ ). (b, d) TOF-MRA images from December 2016 revealing increases in BA tortuosity, elongation (b: length, $46.9 \mathrm{~mm}$ ) and dilation (d: diameter, $10.5 \mathrm{~mm}$ )

progression of dolichoectasia [17]. In the current case study, the patient had developed a BA vessel-wall hematoma and vertebral artery dissection with concurrent obstructive hydrocephalus and severe ischemic strokes. MRA revealed a dilated $\mathrm{BA}$ that extended towards the third ventricle. It may be speculated, therefore, that the development of obstructive hydrocephalus was associated with compression of the foramen of Monro, the cerebral aqueduct, or the third ventricle [18].

The reason for the rapid progression of VBD in the current case is unknown. It is possible that increased blood pressure may have aggravated the natural course of VBD [19]. Hypertension can increase the wall shearing stress of a dolichoectatic BA, leading to the formation of atherosclerosis and, ultimately, to cerebral ischemic stroke. In the presented case, the patient had developed frequent pneumonia due to recurrent posterior circulation ischemic stroke. The occurrence and growth of VBD has been attributed to the interaction between multiple factors, including infection and immune status [1]. A recent study noted that HIV infection was associated with artery adventitial inflammation and dolichoectasia independent of intracranial atherosclerosis, suggesting that differential inflammatory responses may influence the clinical course of intracranial atherosclerosis and dolichoectasia [20]. The post-stroke inflammatory response is the predominant mechanism linking ischemic stroke with infection. In turn, infection can promote atherosclerosis, plaque rupture, and thrombosis, leading to ischemic stroke [21]. Atherosclerosis is often associated with VBD, and may be a pathogenic factor of VBD. An autopsy of a deceased patient who had been diagnosed with an IgG4-related disease revealed some histological features of vertebral arteries and BAs that were very similar to those of an inflammatory aortic aneurysm, suggesting that autoimmune and inflammation mechanisms may also play a role in the etiology of VBD [22]. Therefore, we hypothesize that inflammatory cytokines may promote the fracture or destruction of the inner elastic layer of vessel walls and hyperplasia of intima-media smooth muscle cells, leading to further BA ectasia.

In conclusion, the current case study presents the clinical course of a patient who experienced the full spectrum 
of VBD symptoms within a four-year period. The clinical course, along with a series of CT and MRI studies, demonstrates the evolving process of VBD. Uncontrolled hypertension, repeated infections, and arterial dissection all contributed to the advancement of VBD. Future research should focus on the progression of VBD through multiple modality imaging to gain insight into the underlying pathophysiologic mechanisms of this disease.

\section{Abbreviations}

BA: basilar artery; CBF: cerebral blood flow; CBV: cerebral blood volume; CT: computed tomography; DSC-PWI: dynamic susceptibility contrastenhanced perfusion weighted imaging; DWI: diffusion-weighted imaging; FLAIR: fluid attenuated inversion recovery; FVH: flair vascular hyperintensity; MRC: Medical Research Council; MRI: magnetic resonance imaging; MTT: mean transition time; TOF-MRA: time-of-flight MR angiography imaging; TTP: time to peak; VBD: vertebrobasilar dolichoectasia

\section{Acknowledgements}

We thank the Medjaden Bioscience Limited for their proofreading the manuscript. We also thank the patient's family for their supporting our research.

\section{Funding}

This study was supported by the National Natural Science Foundation of China (81471203).

\section{Availability of data and materials}

All data generated and analyzed in this study are included in this article.

\section{Authors' contributions}

DPZ, HLZ, and MZ conceived of this study, provided financial support and given final approval of the version. DPZ and YFP analyzed the data and drafted the manuscript. QKM and SY performed the analysis and interpretation of imaging data. All authors read and approved the final manuscript.

\section{Ethics approval and consent to participate}

The patient' wife and his family provided a statement of ethics approval and written informed consent. The ethics committee of Zhengzhou People's Hospital approved the study.

\section{Consent for publication}

The patient's wife provided written informed consent for publication of patient clinical details and clinical images.

\section{Competing interests}

The authors declare that the research was conducted in the absence of any commercial or financial relationships that could be construed as a potential competing interest.

\section{Publisher's Note}

Springer Nature remains neutral with regard to jurisdictional claims in published maps and institutional affiliations.

\section{Author details}

'Department of Neurology, The First Affiliated Hospital of Henan University of CM, Zhengzhou, China. ${ }^{2}$ Department of Neurology, People's Hospital of Zhengzhou Affiliated to Southern Medical University, Zhengzhou, China. ${ }^{3}$ Department of Neurology, People's Hospital of Henan Province, Zhengzhou, China. ${ }^{4}$ Department of Image, People's Hospital of Zhengzhou Affiliated to Southern Medical University, Zhengzhou, China.

Received: 4 January 2018 Accepted: 4 April 2018

Published online: 20 April 2018

\section{References}

1. Yuan YJ, Xu K, Luo Q, Yu JL. Research progress on vertebrobasilar dolichoectasia. Int J Med Sci. 2014;11:1039-48.
2. Wolters FJ, Rinkel GJ, Vergouwen MD. Clinical course and treatment of vertebrobasilar dolichoectasia: a systematic review of the literature. Neurol Res. 2013;35:131-7.

3. Lou M, Caplan LR. Vertebrobasilar dilatative arteriopathy (dolichoectasia). Ann N Y Acad Sci. 2010;1184:121-33.

4. Gutierrez J, Sacco RL, Wright CB. Dolichoectasia-an evolving arterial disease. Nat Rev Neurol. 2011;7:41-50.

5. Itabashi R, Mori E, Furui E, Sato S, Yazawa Y, Kawata K, et al. A dilated surface appearance on basiparallel anatomic scanning-magnetic resonance imaging is a useful tool for the diagnosis of spontaneous vertebral artery dissection in lateral medullary infarction. J Stroke Cerebrovasc Dis. 2014;23: 805-10

6. Forster A, Kerl HU, Wenz H, Mürle B, Habich S, Groden C. Fluid attenuated inversion recovery vascular hyperintensities possibly indicate slow arterial blood flow in vertebrobasilar dolichoectasia. J Neuroimaging. 2015;25:608-13.

7. Smoker WR, Corbett JJ, Gentry LR, Keyes WD, Price MJ, McKusker S. Highresolution computed tomography of the basilar artery: 2 . Vertebrobasilar dolichoectasia: clinical-pathologic correlation and review. AJNR Am J Neuroradiol. 1986;7:61-72.

8. Passero SG, Rossi S. Natural history of vertebrobasilar dolichoectasia. Neurology. 2008;70:66-72.

9. Pico F, Labreuche J, Amarenco P. Pathophysiology, presentation, prognosis, and management of intracranial arterial dolichoectasia. Lancet Neurol. 2015; 14:833-45.

10. Samim M, Goldstein A, Schindler J, Johnson MH. Multimodality imaging of vertebrobasilar dolichoectasia: clinical presentations and imaging spectrum. Radiographics. 2016;36:1129-46.

11. Zis $\mathrm{P}$, Fragkis $\mathrm{S}$, Lykouri $\mathrm{M}$, Bageris I, Kolovos $\mathrm{G}$, Angelidakis $\mathrm{P}$, et al. From basilar artery dolichoectasia to basilar artery aneurysm: natural history in images. J Stroke Cerebrovasc Dis. 2015;24:e117-9.

12. Del Brutto VJ, Ortiz JG, Biller J. Intracranial arterial dolichoectasia. Front Neurol. 2017;8:344.

13. Passero SG, Calchetti B, Bartalini S. Intracranial bleeding in patients with vertebrobasilar dolichoectasia. Stroke. 2005;36:1421-5.

14. Matsukawa H, Shinoda M, Fujii M, Uemura A, Takahashi O, Niimi Y. Basilar dolichoectasia and the spontaneous intradural vertebral artery dissection. Brain Inj. 2016:30:90-4.

15. Kim HS, Kwak HS, Hwang SB, Chung GH. Rapid progression of symptomatic vertebrobasilar artery dissection on magnetic resonance imaging: a case report. Neurointervention. 2014;9:106-8.

16. Ahn SS, Kim BM, Suh SH, Kim DJ, Kim DI, Shin YS, et al. Spontaneous symptomatic intracranial vertebrobasilar dissection: initial and follow-up imaging findings. Radiology. 2012;264:196-202.

17. Nakatomi H, Segawa H, Kurata A, Shiokawa Y, Nagata K, Kamiyama H, et al. Clinicopathological study of intracranial fusiform and dolichoectatic aneurysms insight on the mechanism of growth. Stroke. 2000:31:896-900

18. Zisimopoulou V, Ntouniadaki A, Aggelidakis P, Siatouni A, Gatzonis S, Tavernarakis A. Vertebrobasilar dolichoectasia induced hydrocephalus: the water-hammer effect. Clin Pract. 2015;5:749

19. Ikeda K, Nakamura Y, Hirayama T, Sekine T, Nagata R, Kano O, et al. Cardiovascular risk and neuroradiological profiles in asymptomatic vertebrobasilar dolichoectasia. Cerebrovasc Dis. 2010;30:23-8.

20. Gutierrez J, Menshawy K, Gonzalez M, Goldman J, Elkind MS, Marshall R, et al. Brain large artery inflammation associated with HIV and large artery remodeling. AIDS. 2016;30:415-23.

21. Fugate JE, Lyons IL, Thakur KT, Smith BR, Hedley-Whyte ET, Mateen FJ. Infectious causes of stroke. Lancet Infect Dis. 2014:14:869-80.

22. Toyoshima Y, Emura I, Umeda Y, Fujita N, Kakita A, Takahashi H. Vertebral basilar system dolichoectasia with marked infiltration of lgG4-containing plasma cells: a manifestation of IgG4-related disease? Neuropathology. 2012; $32: 100-4$ 